\title{
COVID-19 and psychological assessment teaching practices - Reflections from a South African university
}

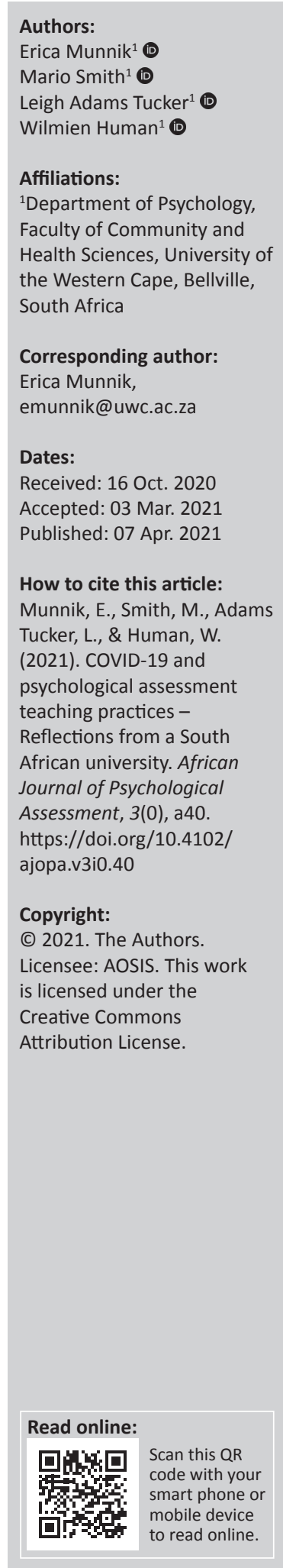

The coronavirus disease 2019 (COVID-19) crisis posed new challenges in higher education, which compounded the existing challenges. The South African higher education sector responded with plans to secure the learning and teaching mandates and bolster support services for students. An emergency remote learning and teaching plan was launched to mitigate the impact of the pandemic in the 2020 academic year. This article reports on the reflections of lecturers who were teaching psychometric assessment and supervising student psychologists on the clinical master's programme during the pandemic. The Master's Clinical Psychology Programme at the University of the Western Cape was the case study. The focus on the psychological assessment module was the unit of analysis. Course documents and reflective notes, generated during the adaptation of psychological assessment training, were used as the data source. Thematic analysis generated five themes, namely, (1) the importance of statutory guidelines for clinical training, (2) adapting content, (3) pedagogy and modalities, (4) management of test libraries and (5) lecturer experience. The management of changes to the module in response to the COVID-19 crisis was challenging. Lecturers had to balance competency training and assessment with revised work and adapted teaching conditions. Emergency teaching interventions took place in the framework of ethics and professional requirement, and the learning outcomes articulated within the scope of practice for clinical psychologists.

Keywords: case study; COVID-19; clinical psychology; learning and teaching; pedagogy; psychological assessment.

\section{Introduction}

Continuous changes in the academic landscape and student protests have been the realities of higher education in South Africa over the past two decades. The higher education sector is considered 'high stress' as functions are performed in a volatile, uncertain, complex and ambiguous environment (Simons, Munnik, Frantz, \& Smith, 2019). The coronavirus disease 2019 (COVID-19) crisis posed new challenges that compound existing socio-structural barriers. President Ramaphosa issued a state of national disaster (Ramaphosa, 2020a), calling for a national lockdown from 23 March 2020 (Ramaphosa, 2020b). This containment response comprised five increasing levels of restriction and prohibition to curb the spread of the virus (South African Government, 2021). The Minister of higher education, science and innovation in South Africa announced an early recess for institutions of higher learning on 17 March 2020 (Nzimande, 2020a). The initial 3-week lockdown at the highest restriction level (5) was extended by 2 weeks. The higher education sector appealed to the South African government to save the 2020 academic year, and institutions developed business continuity plans to this end. Institutional differences related to the historical influences on institutional status and functioning were key in formulating a response to the crises. Thus, it was important to reflect on responses at an institutional level.

The University of the Western Cape (UWC) launched an emergency remote learning and teaching plan to curtail the impact of the pandemic on the 2020 academic year (Lawack, 2020). Staff worked remotely using a range of digital platforms to continue learning and teaching. However, the information and communication technology (ICT) readiness of the country proved a concern through lack of access to sufficient data, reliable internet connectivity and appropriate devices (Department of Health, 2020). Governance and administration continued within the limitations of the business continuity plans, remote working conditions and infrastructure. The phased return of students to university campuses and to work-integrated learning was contingent on the relaxation of containment measures (Nzimande, 2020b). 


\section{Professional training guidelines}

During the lockdown, professional programmes prioritised the completion of theoretical components. With the advent of COVID-19, the guidelines for teaching psychometrics at higher institutions of learning and the minimum standards for training of clinical psychology published by the Board of Psychology (HPCSA, 2019a, 2019b) still served as the principal documents to orientate training towards the requisite professional competencies in psychometric assessment. These competencies included, but were not limited to, the knowledge of psychometric theory; understanding of scientific, theoretical, empirical and contextual bases of assessment; and the skills and techniques to conduct assessments and assess outcomes. Other important competencies include the ability to evaluate multiple roles, contexts and relationships within which client's function, and the ability to understand the collaborative relationship in assessment. Competence in assessment modalities entails a high level of integration and abstraction (HPCSA, 2019a, 2019b).

The Professional Board for Psychology at the Health Professions Council of South Africa (HPCSA) and the Psychological Society of South Africa (PSYSSA) provided guidelines for the adoption and operationalisation of remote training in psychology. These guidelines encouraged institutions that engage in postgraduate training to provide trainees with reasonable clinical exposure as well as support during this time of national disaster (HPCSA, 2020). It also advocated for developing contingency plans for the delivery of high-quality training to postgraduate students who still met the minimum standards of professional education and training. The Professional Board for Psychology further stipulated that alternative assessment practices need to be considered, practical work needs to be rescheduled as far as possible and video conferencing should be considered as an alternative to traditional face-to-face teaching, assessment and intervention practices (HPCSA, 2020). The curriculum had to incorporate the revised guidelines for training.

\section{Programme pedagogy}

Competency-based training in psychometric assessment included theory, practice and integration. The modes of delivery included face-to-face lectures, work-integrated learning and supervision. Under normal circumstances, changes to the modes of delivery were introduced systematically and gradually. Similarly, learning modalities, activities and platforms were selected to enhance the traditional face-to-face (contact) learning and teaching. The pandemic necessitated an emergency online teaching approach that focused on case-based learning supported by paper-based supervision and integration.

Clinical training in the second and third terms was directly impacted by the lockdown and the lack of access to the physical campus (Padmanabhanunni, 2020). The training collective consists of lecturers and clinical supervisors who reconceptualised the teaching of core competencies and learning outcomes of the programme as well as clinical practical requirements and exposure. This article reports on the operational and pedagogical challenges and solutions experienced and changes effected in the psychometric assessment curriculum for student psychologists during the pandemic.

\section{Method}

\section{Aim}

The aim of this article is to identify the adaptations to teaching of psychometric assessment during COVID-19. It reflects on the rationale and impact of the adaptation of the psychometric curriculum for master's-level student psychologists at a South African University.

\section{Design}

A case study design was used to describe and explore adaptation to psychological assessment teaching practices during COVID-19. The case study method helped to attain an in-depth appreciation of the adaptation to teaching (the issue) in response to COVID-19 (the phenomenon of interest) in its naturalistic, real-life context, that is, professional training at the identified university as recommended by Crowe et al. (2011) and Creswell (2013). The case study design facilitated learning about this phenomenon in a micro-environment as described by Yin (2014). The case study design facilitated the examination and reflection on the adaptation of the psychological assessment curriculum during the COVID-19 pandemic.

\section{Case study site}

The research setting or case study site was UWC. The master's programme in clinical psychology is housed in the Department of Psychology and the Faculty of Community and Health Sciences. The master's training programme is a full-time training programme accredited by the HPCSA and leads to registration as a clinical psychologist (HPCSA, 2018). The psychological assessment module is one of three core modules in the clinical programme. It is a year-long module with a minimum of 2 hours of formal teaching a week. The psychological assessment module incorporates, but is not limited to, training in test construction or psychometric theory, the administration, scoring and interpretation of psychological tests in adult and child practice, neuropsychology and intellectual disability. It has a strong focus on the attainment of clinical skills and competency as per HPCSA recommendation (HPCSA, 2020). The module also includes practical exposure to face-to-face assessments at a UWC-based community clinic and exposure to hospitalbased placements from the second term onwards.

\section{Defining the case}

The psychological assessment module was defined as the case. Successful completion of this module is a prerequisite for achieving the published professional competencies in psychological assessment (HPCSA, 2019a). Guiding 
principles about the pedagogy and operationalising the teaching and assessment practices remain the responsibility of specific programme committees.

Training in psychological assessment is a year-long module that included work-integrated learning, lectures, observation of assessments, simulations of test situations, case studies and testing in real time (Faculty of Community and Health Sciences, 2019). The usual mode of delivery was face-to-face instruction. The training team and the students had ready access to a well-curated psychometric test library that supported teaching and learning. Teaching of psychological assessment at UWC is a collective endeavour, where lecturers are engaged in the teaching and supervision of various components of psychological assessment such as psychometric theory, test administration and report writing.

The training team in psychometry consisted of four lecturers with $\mathrm{PhD}$ qualifications, who were appointed at the rank of senior lecturer (3) and associate professor (1). The lecturers were registered clinical psychologists for $17,14,11$ and 10 years, respectively. Their teaching experience in higher education spanned 23, 12, 9 and 7 years, respectively. Their focused experience of 17, 12, 9 and 7 years, respectively, in teaching psychometrics in professional programmes led to their registration as counsellors (BPsych) and psychologists (M.A. Psychology). Their experience of clinical supervision along with psychometric assessment included 17, 12, 11 and 6 years, which were acquired in professional programmes spanning eight institutions and five internships. Thus, there was a breadth and depth of experience in this teaching cohort.

The training team for psychometry reports to the broader collective at monthly meetings. During the imposed early recess, the training team met more regularly to discuss adaptations and teaching strategies. Minutes of these meetings as well as reflective notes by the staff were compiled in order to track decision-making and dynamic aspects including, but not limited to, personal experiences and collective experiences of the adaptation process. Similarly, course outlines and guidelines for student participation and assessment were revised. All these course documents were compiled into an archive that is stored as evidence for accreditation, quality assurance and compliance purposes. The aim of the case study was to establish how COVID-19 affected the teaching and assessment practices of this module at UWC.

\section{Data collection}

The archive of course documents comprised the source of data for this study. The use of records and documents can be limiting in terms of fit for purpose, completeness and robustness (Creswell, 2013). The course documents were specifically created to record changes in the module and the underlying pedagogical and logistic considerations that enhanced its fit for purpose. The process of generating the documents included reflexive notes generated by clinical teachers in response to stimulus prompts such as 'Did changes occur in teaching and assessment practices during COVID-19?'
'If so why?' and 'In what way did it change?' The reflexive notes provided rich, thick descriptions and abstractions that in turn constituted robust raw data. The documents were generated in the process of adaptation and were used to inform decision-making and provide accountability mechanisms. The resultant documents had a high level of completeness which made it appropriate for the study.

Multiple sources of data such as revised course outlines that accommodated online teaching, revised assessment schedules, instructions to students about the proposed changes to the digital learning management system (iKamva) and lecturer reflections were considered to develop a holistic picture of the data pertaining to the case study. The use of multiple sources of data increased the trustworthiness of the study as recommended by Crowe et al. (2011).

\section{Data analysis}

Thematic analysis was used for data analysis. Two reviewers immersed themselves into the data through repeated reading and viewing of all the sources. Data were organised and coded to allow key issues to emerge. Key issues were organised into themes. The data analysis followed the basic steps outlined by Creswell (2013) for thematic analysis. The analysis was conducted by two people, of which one taught on the module in 2020. The resultant themes were shared with the remaining lecturers on the module in two rounds of reflexive discussions until consensus was reached between the lecturers and the independent analyst. This approximated respondent validation and served to enhance the authenticity and trustworthiness of the results through collective alignment of themes as recommended by Harrison, Birks, Franklin and Mills (2017).

\section{Ethical considerations}

The data were produced as part of an educational quality assurance process. Secondary research constitutes low-risk research and does not require ethics clearance. No students were identified or no work produced by students were used. The data were generated by the authors as the lecturers involved in the quality assurance process. The lecturers consented to the use of the documents for the purposes of producing this manuscript. These documents are available in selected shared digital spaces. The potential benefits of this article include a contribution to the community of practice in professional training during the COVID-19 pandemic. The draft manuscript was presented to the Office of the Deputy Registrar at UWC to obtain permission for publishing an article in which the course documents were used and the institution is named. Through this process, the risks associated with reputational harm to third parties and the institution were mitigated or averted. Written permission for submission of the article was obtained as a letter of endorsement with reference number: UWCEN161020MS on 16 October 2020. Basic principles such as proper anonymisation of data were applied in the study. Only information related to teaching and supervision experience in higher education, placement and qualification were 
disclosed to provide context. This information is available in the public domain and in the register of the HPCSA. The lecturers consented to the content and the nature of information shared in the article.

\section{Results}

The transition to online learning, focused on digitised teaching and learning methods, posed various challenges to achieve the bridging of theory and practice and mastery of clinical competencies. Five themes emerged, namely, (1) guidelines for clinical training, (2) adapting content, (3) pedagogy and modalities, (4) managing test libraries and (5) lecturer experience.

\section{Guidelines for clinical training}

The training team reflected that the responsiveness demonstrated through the provision of revised guidelines was containing personally and facilitative at an operational level. The responsiveness of the professional bodies provided a framework that in turn promoted a common reference and sense of a community of practice. The teaching team could make decisions about adaptation to the curriculum within a clear framework that provided both security and direction during uncertain times.

\section{Adapting content}

There was variation in the ease with which content could be adapted for emergency remote teaching. This variation related to the nature of test contents as well as the structure of the tests.

\section{Nature of content}

Adapting teaching modes was easier for principles of test construction such as validity and reliability. Abstract constructs that underpin tests were more challenging. Theoretical and operational definitions were more readily translated for remote teaching. In contrast, the more nuanced, dimensional understanding of constructs demanded more engagement than the digital platform could afford. Such constructs were further illustrated using clinical examples that drew more heavily on the experience of the lecturer, as students were not exposed to clinical settings. The nature of the content intersected with the extent to which students had clinical references. The domains in cognitive assessment were challenging to teach. Personality assessment was less sensitive to issues of practice effects and teaching during measurement, creating greater flexibility in how the resource could be demonstrated to students.

\section{Type of test}

Self-report measures, for example, structured personality assessments, use questionnaire test booklets with standardised instructions. Test booklets are available in hard copy or digital form. The structured nature of these tests shifts the emphasis of training onto scoring and interpretation of client profiles as administration is relatively uncomplicated and can be selftaught. Such tests allowed for independent work to become familiar with the test and administration. This provided a basis for teaching technical aspects like scoring and higher order aspects such as interpretation. The additional time available to focus on interpretation of structured measures appeared to mitigate the impact of remote teaching and the limited contact.

Projective tests required actual teaching of test administration and scoring to ensure that students understood the highly specified administration procedures and scoring systems in addition to the interpretation and online exposure to manifest the content of stimulus cards. In this instance, the online environment was secured to avoid the risk of violating the confidentiality of content through unauthorised auditing. Thick descriptions of manifest content were provided rather than visual stimuli as part of presentation slides or screen share functions. Teaching the administration and scoring of projective personality tests was done using anonymised protocols. Students were given completed protocols to practice scoring. The uniformity of protocols allowed for comparison between students and mastery was achieved through providing multiple cases to score. The number of completed scoring trials far exceeded the number of students who would have achieved through actual administration.

The teaching of cognitive measures in an online learning space was more challenging as it entailed mastery of complex administration and high-level interpretation. Cognitive and developmental measures require the test administrator's familiarity with various novel test components. Students' limited access to test material during lockdown substantially reduced the opportunity to familiarise themselves with the material. Logistic challenges included access to test materials and challenges of a conceptual nature such as how to teach students to effectively administer, score and interpret tests without face-to-face assessments. Ethical concerns in online teaching such as copyright for material used (digitisation or copying), the confidentiality and the security of test content posed threats during online teaching.

\section{Experiential aspects}

Live assessment was not feasible, given the social distancing and travel restrictions. The clinic on campus remained closed and physical access was only permitted to a selected number of students within the allowed 30\% during the adjusted level 3, for example, access to science labs. Although online counselling services were available, no psychometric assessments were possible. Student psychologists (M1s) were not permitted to access the clinical platform and, therefore, could not visit hospital-based placements. Viable alternatives had to be found to provide experiential learning. Case studies effectively helped to develop clinical reasoning, selection of tests and compilation of batteries. Protocols from the departmental archive provided appropriate training cases that students could use to master scoring and interpretation in an online space without the risk of direct client contact. The remedial learning on administration will take place during internships (HPCSA, 2020). The time allocated for 
supervision was used to focus on learning and integration from case studies and protocols.

\section{Pedagogy and modalities}

Providing epistemological access to the complex nature of psychometric assessment remains a key challenge when teaching. The requirement of high level of technical and interpretive skills is challenging to all students regardless of the prior learning experiences and individual differences in ability. It is also important to hone the skills of observing and describing behaviour during testing in the context of assessment. The ability to read emotional cues during the physical assessment is key for the trainee psychologist to master. This learning was deferred to internship because of continued restrictions that made direct client contact difficult. Similarly, the teaching clinician has a reduced opportunity to use the emotional resonance in the classroom to assess and track students in terms of engagement, comprehension, emotive responses and reactivity to text content as well as the ability to learn and generalise acquired skills.

During remote teaching, students typically disable the video function on their devices in order to save bandwidth and maintain privacy. This further reduces the ability to assess students learning in live, albeit digital, settings. This in turn detracts from the ability to understand where students may struggle, where to provide further elaboration or general encouragement. The virtual classroom blunts this effect and contributes towards feelings of isolation.

The virtual classroom and online formats required additional consideration and actions in order to ensure a safe and ethically sound learning space. Staff had to manage the boundaries of online classrooms by setting passwords that were valid for restricted periods and creating defined participant lists. In this way, the risk of accidental access to confidential content and test material was reduced. Similarly, the end-to-end encryption on platforms maintained the confidentiality of the teaching space.

The online learning environment included a number of modalities. The advantages and disadvantages of each are outlined below.

\section{Narrated PowerPoint slides}

Lecturers compiled slides under the pressure of time during early recess. The quick turnaround period to revise or prepare the slides in the required format was challenging and largely dependent on the level of technical skill in the teaching complement. Pre-recorded narrated presentations were supplemented with worksheet exercises and online question and answer sessions. Completion of the narrated slides did not equate to the confidence that the work was effectively covered to achieve learning, mastery and integration. Tracking students' downloading of prescribed material and narrated slides did not sufficiently replace tracking engagement with the content. Narrated PowerPoint slides gave students flexibility to work through the material at their own time relative to the competing demands of studying from home. Data vulnerability and connectivity were obstacles to students' ability to access and engage the material.

\section{Social media applications}

Applications such as WhatsApp provided a platform for contact between the staff and the students. Student queries, quick announcements and 'checking in' were easily facilitated via this platform in a data-light manner. It proved to be useful to foster open communication and greater access and connection with students. In this way, it created a community of practice with the flexibility to respond even whilst otherwise occupied, for example attending a meeting. This format required explicit boundary setting and underscoring professional use of the platform to make it fit for the purpose when connecting via personal devices.

\section{Webinars}

Webinars presented as 90-min sessions were used as critical discussion forums after students worked through relevant content distributed through the secure online university learning management platform. These sessions facilitated 'check-ins' both to hear how students were doing and to identify the challenges in learning.

\section{Video recordings}

Video recordings of demonstrations of test administration were useful to offset the loss of contact sessions where demonstrations would have normally taken place. The recordings become resources that students can revisit if necessary. This contributed to deepened understanding and fostered learning. The management and regulation of access to such video materials were critical to ensure that the ethical obligation of keeping the content of test material confidential is met. Thus, the instruction in professional and ethical conduct had to be explicit, and measures are introduced to formalise the ethical obligations for students, for example a confidentiality binding agreement. Although these measures cannot guarantee that transgressions will not occur, it provides a clear account of ethical conduct in the teaching and instructional space, which confirms that the student was appropriately and properly informed. Such documented processes can be subsequently used in disciplinary processes.

\section{Case studies}

Case studies provided an alternative to practical hands-on training during lockdown. Aspects like scoring, interpretation and report writing were facilitated through the use of case studies to integrate the various components of assessment. History taking, compilation of test batteries, observation of behaviour during testing and clinical reasoning required additional facilitation in order to develop mastery from case study methods. The former set of skills could be developed to a greater extent whilst the latter set of skills was achieved to a lesser extent than during the normal circumstances. 
During the pandemic, the transition to online learning was an emergency measure implemented under the pressure of time with the possibility of contact being uncertain and unconfirmed. The pedagogy, teaching practices and assumptions underpinning the psychological assessment curriculum was re-examined, evaluated and adapted with formats and modes of delivery not typically associated with professional training in general and psychometric assessment in particular. The challenge was to ensure that adaptations resulted in an inclusive learning experience. The resultant curriculum of necessity had to be dynamic, responsive to changing demands and sensitive to contextual realities of students in South Africa. Contextual challenges such as load shedding, unequitable access to data and devices, socioeconomic challenges and the extent to which home environments were conducive to living and learning, increased family responsibilities during lockdown and stereotypical gender roles and division of labour had to be considered.

\section{Managing test libraries}

Professional training in psychometry requires access to a secure and well-managed test library. As mentioned before, the Psychology Department at UWC has a well-curated test library that ensures access to current test material, despite the resource constraints associated with a historically disadvantaged institution. ${ }^{1}$ During the stringent lockdown, this resource was not accessible. During subsequent relaxation of the measures, the resource became accessible, but practical contact with clinical cases or referrals was still not possible because of the continuation of social distancing and the risk that close contact posed for infection. Duplication of material was allowed for teaching purposes but posed greater challenges as the appropriate storage and management of such copies could not be ensured. Thus, it was not considered feasible. The development of teaching videos became an extension of the test library subject to the same controls and regulation for storage and access. As mentioned before, this necessitated explicit instruction in and agreements about management of the said materials. Thus, achieving the learning outcomes during the pandemic posed challenges to the conceptualisation and implementation of the rules for managing the confidentiality of and access to test materials.

\section{Lecturer experience}

As described earlier, the breadth and depth of experience in the training collective was substantial. Lecturers were able to draw on their teaching, supervisory and clinical experience to offset limitations in the current teaching context. The ability to provide real-life clinical references assisted students to develop a more nuanced understanding of key constructs in assessment. The depth in teaching experience across programmes and institutions provided a strong base from which to move into adaptation. Thus, the confidence in the content and a clear understanding of the learning outcomes and competencies that had to be mastered meant that more attention could be focused on finding alternate modes of teaching and creating space for reflective practice. The depth of experience in supervision of psychometric assessment articulated into a good understanding of how to work developmentally and to facilitate the learning needs of students. In the adaptation process, this experience was important as it provided a framework and a reference for decision-making about which aspects to prioritise and which aspects to defer. The breadth and depth of experience was as important as the intentional reflexive practice in the programme, which meant that the training team was able to access the experience and had the disposition to want to access it.

\section{Discussion}

The COVID-19 pandemic necessitated emergency responses from the higher education sector in order to ensure business continuity and the completion of the 2020 academic year. In the context of professional programmes, such as clinical psychology, adaptations and accommodations had to make educational sense and satisfy professional competency and statutory requirements. The pandemic challenged the pedagogy and learning principles, underpinning professional training in psychometric assessment. Course adaptations had to be contextually appropriate considering the physical, emotional, social and financial barriers students would potentially face during COVID-19. The findings identified that the ability to be responsive and make meaningful changes was in part facilitated by clear frameworks from the professional bodies. The professional bodies provided an adapted statutory framework that guided curriculum changes in psychometric assessment. Decisions about adaptations to curriculum were taken within this adapted framework. Careful, albeit pressured, considerations of the learning outcomes and competency requirements assisted in theidentification and implementation of realistic amendments to the curriculum within the broader competency framework provided by professional bodies. This was consistent with the literature on curriculum adaptation and re-curriculisation (Barab \& Luehmann, 2003; Brown, 2009). Familiarity with the professional frameworks and minimum competency requirements for psychometric assessment practices was a facilitator of responsiveness and meaningful adaptation of the curriculum in the context of emergency response to the pandemic.

The findings indicated that the conceptualisation of professional training as a process involving different phases and stakeholders promoted responsiveness. Such a conceptualisation allowed the training team to identify the skills that could be deferred to internship. The training in the first year of the programme could then prioritise mastery of theoretical concepts and baseline clinical competencies that were deemed essential for the completion of first year clinical training in psychometry and progression to internship. In this way, the training could ensure that adapted learning outcomes and competency requirements were attained. This resonates with the HPCSA's policy regarding intern 
psychologists, guidelines for universities, internships, training institutions and intern psychologists (2015) who underscored the importance and value of conceptualising clinical training as a cumulative and continuous process that spans theoretical training, internship and community service. The training offered in psychometric assessment in the theoretical component of the programme must be understood in the broader context of clinical training. The complementary relationship between course work, internship and supervised practice enabled decision-making about adaptations to and the timing of teaching clinical competencies. The practical value of the internship as an extension of training in assessment was underscored.

The literature indicated that universities with the existing ICT infrastructure and learning management systems were able to migrate faster to emergency remote teaching using digital platforms (Almaiah, Al-Khasawneh, \& Althunibat, 2020; Crawford et al., 2020; Eltahir, 2019; Shehzadi et al., 2020; UWC, 2020). The findings of the current study further illustrated this point. The existence of digital learning management systems at the institution facilitated an easier transition to online learning with a strong digital or online focus.

This study demonstrated that the responsiveness of institutions was influenced by their governance structures. The identified institution was able to provide flexible governance and decisive leadership in academic planning and quality (UWC, 2021a, 2021b, 2021c). The readiness and responsiveness of quality assurance processes at the institution enabled the amendments to assessment schedules and curriculum changes. Flexible governance reduced pressure on the system in all respects and reflected in the lecturer experiences.

The findings illustrated that adaptation to teaching practices and modes of learning was linked to the type of assessment measure and the nature of the content. Theoretical concepts, psychometric properties and self-administered measures were easily taught. It required less focused instruction in and practice of administration that in turn freed up time to focus on scoring, interpreting and report writing. Projective personality tests, cognitive and developmental measures required explicit instruction in administration in addition to scoring, interpreting and report writing. Immersion in reallife assessments was not possible, and the requisite skills were taught through case-based learning. The availability of a well-curated archive of developmentally appropriate cases including original protocols assisted the shift to case-based learning. Similarly, the creative use of instructional videos, narrated slides, simulation and digital classrooms were useful to teach assessment. Teaching in a collective also provided a community of practice and sound boarding that reduced the isolation of teaching in this context. The depth in the experience of the teaching staff in psychometric assessment and clinical supervision contributed to the readiness and capacity to be adaptive. Reflexive practice and team-based training positively influenced the process of curriculum adaptation.

Teaching psychometric assessment during the pandemic necessitated additional considerations in the ethical management of test materials. The findings illustrated the importance of regulating the use of test content and materials in slide presentations (HPCSA, 2019a, 2019b). In addition, it was necessary to secure the digital classroom to prevent unauthorised access and auditing. The recording of lectures and instructional videos became useful resources and required a binding agreement around the use and storage as it constituted a part of the test library.

\section{Limitations of the study}

One of the key limitations of the study is that it focused on one of the three professional programmes in psychology offered at the institution, despite the rationale provided for the decision. The study does not include student reflections and thus is limited in terms of the insights into the subjective experiences of students of this revised programme. Similarly, the article and study focused on the operational and pedagogical issues experienced by staff whilst not addressing the subjective experience of working through re-conceptualisation. The demographic information of the lecturers involved was not provided in order to protect identities, and it was not considered important for the collective-level data. In this way, some of the dynamic information about the relative position of the lecturers in the collective is lost to analysis. Such an analysis, although useful, was outside the scope of what this manuscript set out to do.

\section{Concluding remarks}

The present case study is reflective of the many challenges and considerations that COVID-19 posed and necessitated in the training of clinical psychologists in psychometric assessment at higher education institutions. It demonstrates how learning and teaching were adapted and re-aligned within the framework of statutory and professional requirements to facilitate a digital online environment for students to still attain professional competency in psychological assessment.

\section{Acknowledgements Competing interests}

The authors declare that they have no financial or personal relationships that may have inappropriately influenced them in writing this article.

\section{Authors' contributions}

E.M. and M.S. participated in the conceptualisation, design, composition of the study and writing of the manuscript. All the authors participated in the reflections, preparation and critical revision of the manuscript. 


\section{Funding information}

This research received no specific grant from any funding agency in the public, commercial, or not-for-profit sectors.

\section{Data availability}

The source of data was confidential course documents that are not available for public distribution.

\section{Disclaimer}

This research has not been commissioned nor does it represent any affiliated agency of the authors.

\section{References}

Almaiah, M.A., Al-Khasawneh, A., \& Althunibat, A. (2020). Exploring the critical challenges and factors influencing the e-learning system usage during COVID-19 pandemic. Education and Information Technologies, 25, 5261-5280. https://doi. org/10.1007/s10639-020-10219-y

Barab, S.A., \& Luehmann, A.L. (2003). Building sustainable science curriculum Acknowledging and accommodating local adaptation. Science Education, 87(4), 454-467. https://doi.org/10.1002/sce.10083

Brown, M.W. (2009). The teacher-tool relationship. Mathematics teachers at work: Connecting curriculum materials and classroom instruction (pp. 17-36). New york, NY: Taylor and Francis

Crawford, J., Butler-Henderson, K., Rudolph, J., Malkawi, B., Glowatz, M., Burton, R. ... Lam, S. (2020). COVID-19: 20 countries' higher education intra-period digita pedagogy responses. Journal of Applied Learning and Teaching, 3(1), 1. https:// doi.org/10.37074/jalt.2020.3.1.7

Creswell, J.W. (2013). Qualitative inquiry and research design: Choosing among five approaches. Thousand Oaks, CA: Sage.

Crowe, S., Cresswell, K., Robertson, A., Huby, G., Avery, A., \& Sheikh, A. (2011). The case study approach. BMC Medical Research Methodology, 11(1), 100. https:// doi.org/10.1186/1471-2288-11-100

Department of Health. (2020). Minister of higher education, science and innovation. Statement on the measures to phase out the lockdown and phasing in of PSET strategic functions. 30 April 2020. COVID 19. Online resource and news porthole. Retrieved from https://sacoronavirus.co.za/2020/04/30/minister-of-highereducation-science-and-innovation-statement-on-the-measures-to-phaseout-thelockdown-and-phasing-in-of-pset-strategic-functions

Eltahir, M.E. (2019). E-learning in developing countries: Is it a panacea? A case study of Sudan. IEEE Access, 7, 97784-97792. https://doi.org/10.1109/ ACCESS.2019.2930411

Faculty of Community and Health Sciences. (2019). Prospectus: Department of Psychology University of the Western Cape. Retrieved from https://www.uwc. ac.za/Faculties/CHS/Psychology/Pages/Academic-Programmes.aspxon

Harrison, H., Birks, M., Franklin, R., \& Mills, J. (2017). Case study research: Foundations and methodological orientations. Forum Qualitative Sozialforschung/Forum: Qualitative Social Research, 18, 1.

Health Professions Council of South Africa (2015). Policy regarding intern psychologists, guidelines for universities, internships training institutions and intern psychologists. (Form 160). Retrieved from https://www.hpcsa.co.za/Uploads/PSB_2019/ Policy\%20and\%20Guidelines/Form\%20160\%2028\%20\%20\%20\%20updated-\%20 October\%202014\%20Final.pdf
Health Professions Council of South Africa. (2018). Professional board for psychology: List of accredited universities in South Africa. Retrieved from https://www.hpcsa. List of accredited universities in South Africa. Retrieved from https://www.hpcsa.
co.za/Uploads/PSB_2019/ACCREDITED $\% 20$ UNIVERSITIES $\% 20 I N \% 20$ SOUTH $\% 20$ co.za/Uploads/PSB_201

Health Professions Council of South Africa. (2019a). Guidelines for the teaching of psychometrics at higher institutions of learning. The Professional Board for Psychology. Retrieved from https://www.hpcsa.co.za/Uploads/PSB 2019/ Psychology. Retrieved from https://www.hpcsa.co.za/Uploads/PSB_2019/
Guidelines $\% 20$ for $\% 20$ the $\% 20$ teaching $\% 20$ of $\% 20$ psychometrics $\% 20 F i n a l . p d f$

Health Professions Council of South Africa. (2019b). Minimum standards for the training of clinical psychology. The Professional Board for Psychology. Retrieved from https://www.hpcsa.co.za/Uploads/PSB_2019/Policy\%20and\%20Guidelines/ SGB\%20CLIN\%20-\%20Revised\%200ctober\%202019.pdf

Health Professions Council of South Africa. (2020). Covid 19 guidelines. Retrieved from https://www.psyssa.com/hpcsa-covid-19-guidelines/

Lawack, V. (2020). No student will be left behind. 15 April 2020. University of the Western Cape. Retrieved from https://www.uwc.ac.za/Announcements/Pages/ No-Student-Will-Be-Left-Behind.aspx

Nzimande, B. (2020a). Address by Minister of Higher Education, Science and Innovation, on measures to deal with the COVID-19 threat in the post-school education and training sector (17/03/2020). The Presidency, South African Government. Retrieved from https://www.polity.org.za/article/sa-blade-nzimande-address-by-minister-ofhigher-education-science-and-innovation-on-measures-to-deal-with-the-covid-19threat-in-the-post-school-education-and-training-sector-17032020-2020-03-20

Nzimande, B. (2020b). Minister of Higher Education, Science and Innovation: Statement on progress in the implementation of measures by the post school education sector in response to covid-19 epidemic. 09 June 2020. Retrieved from https://www.gov.za/speeches/minister-blade-nzimande-progressimplementation-coronavirus-covid-19-measures-post-school

Padmanabhanunni, A. (2020). Personal communication by course coordinator to the Masters Psychology collective. Psychology Department. Cape Town: University of the Western Cape.

Ramaphosa, C. (2020a). Measures to combat Coronavirus COVID-19 epidemic The Presidency, South African Government. Retrieved from https://www.gov.za/ The Presidency, South African Government. Retrieved from https://www.gov.za/
speeches/statement-president-cyril-ramaphosa-measures-combat-covid-19speeches/statement-president
epidemic-15-mar-2020-0000

Ramaphosa, C. (2020b). Escalation of measures to combat Coronavirus COVID-19 pandemic. The Presidency, South African Government. Retrieved from https:// www.gov.za/speeches/president-cyril-ramaphosa-escalation-measures-combatwwW.gov.za/speeches/president-cyril-r
coronavirus-covid-19-pandemic-23-mar

Shehzadi, S., Nisar, Q.A ., Hussain, M.S., Basheer, M.F. Hameed, W.U, \& Chaudhry, N.I. (2020). The role of digital learning toward students' satisfaction and university
brand image at educational institutes of Pakistan: a post-effect of COVID-19. Asian Education and Development Studies, https://doi.org/10.1108/AEDS-04Asian Educati $2020-0063$

Simons, A., Munnik, E., Frantz, J., \& Smith, M. (2019). The profile of occupational stress in a sample of health profession academics at a historically disadvantaged university in South Africa. South African Journal of Higher Education, 33(3), university in South Africa. South African Journ
132-154. https://doi.org/10.20853/33-3-3199

South African Government. (2021). Covid-19 alert systems. Retrieved from https:// www.gov.za/covid-19/about/about-alert-system

University of the Western Cape. (2021a). Flexible learning and teaching provisioning policy draft. Academic Planning Unit (APU). Cape Town: University of the Western Cape.

University of the Western Cape. (2021b). Centre for Innovative Education and Communication Technologies (CIECT). Student orientation interventions. Cape Town: University of the Western Cape.

University of the Western Cape. (2021c). Communication. From the Office of the Executive Director: Human Resources - Update on Covid-19 protocols. Cape Town: University of the Western Cape.

University of the Western Cape Communication. (2020). Workshop and training material now online and on iKamva. Division for Postgraduate Studies. Cape Town: University of the Western Cape.

Yin, R.K. (2014). Case study research: Design and methods. Los Angeles, CA: Sage. 